\title{
Evaluation of Copan FLOQSwab for the molecular detection of Chlamydia trachomatis by Abbott RealTime CT PCR
}

\author{
Liselotte Coorevits ${ }^{1}$, Canelle Vanscheeuwijck ${ }^{2}$, Ans Traen*2, Luk Bingé ${ }^{2}$, \\ Inge Ryckaert ${ }^{1}$, Elizaveta Padalko ${ }^{1,3,4}$
}

${ }^{1}$ Department of Laboratory Medicine, Ghent University Hospital, Belgium, ${ }^{2}$ Pasop VZW, Belgium, ${ }^{3}$ Department of Clinical Chemistry, Microbiology and Immunology, Ghent University, Belgium, ${ }^{4} S$ chool of Life Sciences, Hasselt University, Diepenbeek, Belgium

\begin{abstract}
Objectives: We evaluated Copan FLOQSwabs next to Abbott swabs for the detection of Chlamydia trachomatis (CT) by Abbott RealTime PCR.

Methods: We collected 1062 paired swabs from female sex workers. The study was divided in two arms, according to the order of swab collection.

Results: If the Abbott swab was collected first, 501 couples were concordant and two discordant (Abbott negative and Copan positive). If the Copan swab was collected first, 537 couples were concordant and 10 discordant (eight Abbott negative and Copan positive and two Abbott positive and Copan negative). All discordant samples contained low levels of $C$. trachomatis. Technical issues lead to retesting of 64 Copan and 21 Abbott swabs.

Conclusion: Our results show that Copan FLOQSwabs can be used interchangeably with Abbott swabs. While appearing to have an advantage in detecting more positive samples, the use of Copan swabs led to a higher retesting rate due to technical errors.
\end{abstract}

Keywords: Molecular techniques, Sexual health, Chlamydia trachomatis

\section{Introduction}

Sexually transmitted infections (STIs) are a major public health concern. Worldwide, Chlamydia trachomatis (CT) is the most common bacterium responsible for STIs. Risk factors include high frequency of partner change, multiple partners, unprotected sex and being unmarried. ${ }^{1}$ In the USA, $C$. trachomatis continues to be the most commonly reported notifiable disease. Remarkably, in 2013, the rate of reported cases decreased for the first time since the start of national reporting in $1993 .^{2}$ In Europe, an overall increasing trend in the number of reported C. trachomatis cases was seen from 1990 to 2009. Since then, the overall rate has slightly decreased, with the main reason being that Bulgaria started reporting very low rates of $C$. trachomatis. $^{3}$ Belgian passive surveillance systems for $C$. trachomatis show a continuous increase in incidence since $2002 .^{4}$ It is presumed that those data are even

Correspondence to: Liselotte Coorevits, Department of Laboratory Medicine, Ghent University Hospital, De Pintelaan 185, 9000 Ghent, Belgium. Email: liselotte.coorevits@ugent.be

*Both authors contributed equally. underestimated due to dependence on the quality of STI services, the intensity of case finding and diagnosis, the quality of reporting and the extent to which patients seek health care. Indeed, a large number of STIs are asymptomatic and only part of the symptomatic population consult a physician. If left untreated, $C$. trachomatis infections can lead to severe complications, mainly in young women, such as pelvic inflammatory disease, ectopic pregnancy and tubal factor infertility. ${ }^{5}$ The Centre for Disease Control and Prevention (CDC) guidelines call for routine screening of all sexually active women between ages 15 and 24, irrespective of symptoms. The rationale is triple: most genital $C$. trachomatis infections are asymptomatic; C. trachomatis prevalence is the highest in this group; $C$. trachomatis related complications can be prevented by screening and treatment of those infected. ${ }^{6}$

A variety of nucleic acid amplification tests (NAATs) to detect $C$. trachomatis in (self-collected) urethral, urine, vaginal and cervical specimens are currently available with far higher sensitivity than previously available diagnostic tests, such as culture, antigen detection and nucleic acid hybridisation. 
However, concerns have raised about their specificity, particularly when screening low-prevalence populations. Indeed, some studies could not reproduce positive NAAT results. ${ }^{6-11}$ Those findings led the CDC to recommend confirmatory testing of positive NAAT results with a second NAAT (with equal sensitivity) when the positive predictive value (PPV) is below $90 \%{ }^{6,12}$ Another significant concern with regard to the performance of NAATs has been the cellular adequacy of the cervical specimen, since $C$. trachomatis is an intracellular organism, targetting the columnar epithelial cells. To assure efficient (self-)sample collection, a variety of rayon, cotton and flocked swabs have been developed, including the swabs developed by the manufacturer of the NAAT. This variety has a beneficial impact on the accessibility of STI testing for healthcare providers.

In Belgium, an outreach health programme targetting sex workers has been set up for the Flemish region, which is named PASOP. Within this programme, the health team offers screening for STIs, a cervical smear and vaccination against hepatitis B. C. trachomatis testing is performed on first contact as part of basic STI screening, after a perceived risk (e.g. condom breakage) or when signs and symptoms are present. Those positive are treated or referred for treatment and the importance of partner treatment is emphasised. ${ }^{13}$ The PASOP population was the subject of our study. The first objective was to evaluate the interchangeability of Copan FLOQSwabs (Copan Italia, Brescia, Italy) and the manufacturer recommended Abbott swabs (Abbott Laboratories, Illinois, USA) for the subsequent detection of $C$. $t r a-$ chomatis by Abbott RealTime $C$. trachomatis PCR in vaginal samples of 1062 Belgian sex workers. Second, we wanted to investigate whether the use of flocked swabs improved the detection of $C$. trachomatis in this population.

\section{Methods}

The population of the current study consists of female sex workers, embedded in an outreach health programme for sex workers in Flanders (PASOP). Between April 2011 and January 2013, each female provided vaginal samples for the detection of C. trachomatis. A couple of two swabs were consecutively collected: an Abbott swab and a Copan FLOQSwab. Each swab was transported individually in Abbott collection medium, and samples were stored at $\left(20^{\circ} \mathrm{C}\right.$ prior to analysis. In the first half of the study, from 6th April 2011 to 23rd February 2012, the Abbott swab was collected before the Copan swab $(n=509)$. From 24th February 2012 to 4th January 2013, the Copan swab was the first one collected, followed by the Abbott swab $(n=553)$. Samples were tested for
C. trachomatis by the Abbott RealTime C. trachomatis PCR on m2000sp and m2000rt instruments, according to the manufacturer's instructions. This assay reports a qualitative result (negative or positive) as well as DC-value, indicative for the bacterial load. The DC-value is the difference between the cycle threshold $(\mathrm{Ct})$ of the internal cutoff control and the $\mathrm{Ct}$ of the sample. The higher the bacterial load is, the larger the difference between both Cts and the higher the DC. Each sample was retested in the case of a failed internal control (internal control error) or a $C$. trachomatis bacterial load below the limit of quantification (beyond cutoff error), whenever enough sample was available. As the PASOP population concerns anonymised subjects receiving preventive healthcare services on a voluntarily basis and are not connected to the Ghent University Hospital whatsoever, no ethical clearance was submitted at the ethical committee of the Ghent University Hospital.

\section{Results}

During 21 months, a total of 1062 couples of swabs were collected from female sex workers, embedded in a Belgian outreach health programme. The study was divided in two arms, according to the order of swab collection. The results of the $C$. trachomatis analyses are presented in Fig. 1. If the Abbott swab was collected first, 32 coupled samples were both positive, 446 both negative and 31 samples needed retesting. Due to exhaustion of the specimen's volume, retesting was impossible in 6/31 samples. Of the remaining 25 samples, one was positive concordant, 20 were negative concordant, two showed an $\mathrm{A}-\mathrm{C}+$ discordance (negative on the Abbott swab and positive on the Copan swab) and two were to be retested, both finally negative concordant. If the Copan swab was collected first, 32 coupled samples were both positive, 478 both negative, three were $\mathrm{A}-\mathrm{C}+$ discordant and 40 needed retesting. There was sufficient sample for retesting in 35/40 samples, of which three were positive concordant, 23 were negative concordant, three were $\mathrm{A}-\mathrm{C}+$ discordant, one was $\mathrm{A}+\mathrm{C}-$ discordant (positive on the Abbott swab and negative on the Copan swab) and five were to be retested. As one sample was yet fully used, only $4 / 5$ could be retested, of which one was positive concordant, two showed an $\mathrm{A}-\mathrm{C}+$ discordance and one showed an $\mathrm{A}+\mathrm{C}-$ discordance. When comparing DC values of concordant positive samples, a statistical difference was observed ( $P$-value 0.00028 first arm; $P$-value 0.00015 second arm) with analyses on Copan swabs systematically resulting in higher DC values, indicating the better sensitivity (Fig. 2). 

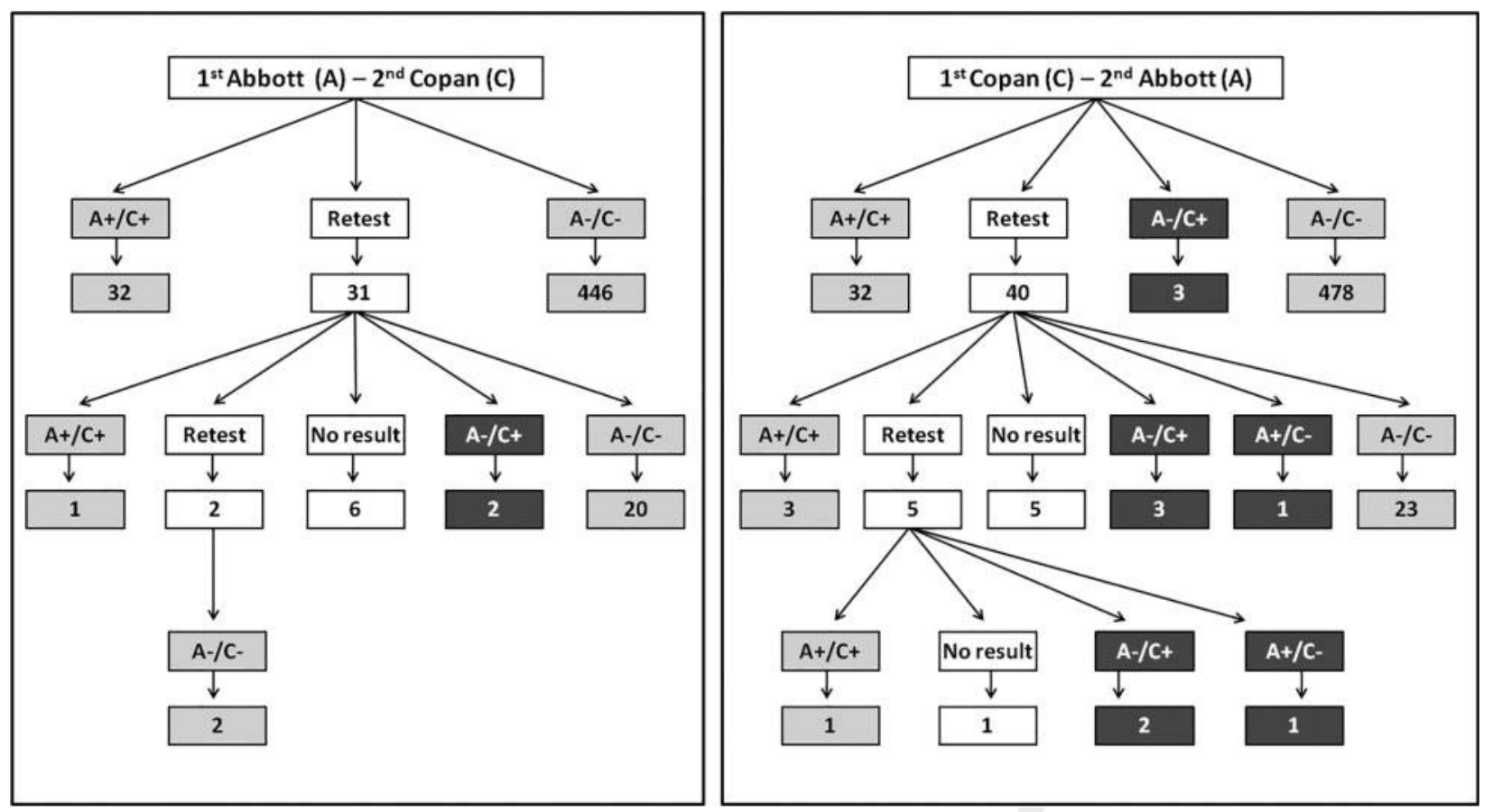

Figure 1 Overview of the results for each study arm. Left panel: Abbott swab collected before Copan swab. Right panel: Copan swab collected before Abbott swab (light grey background: concordance between both swabs; dark grey background: discordance between both swabs).

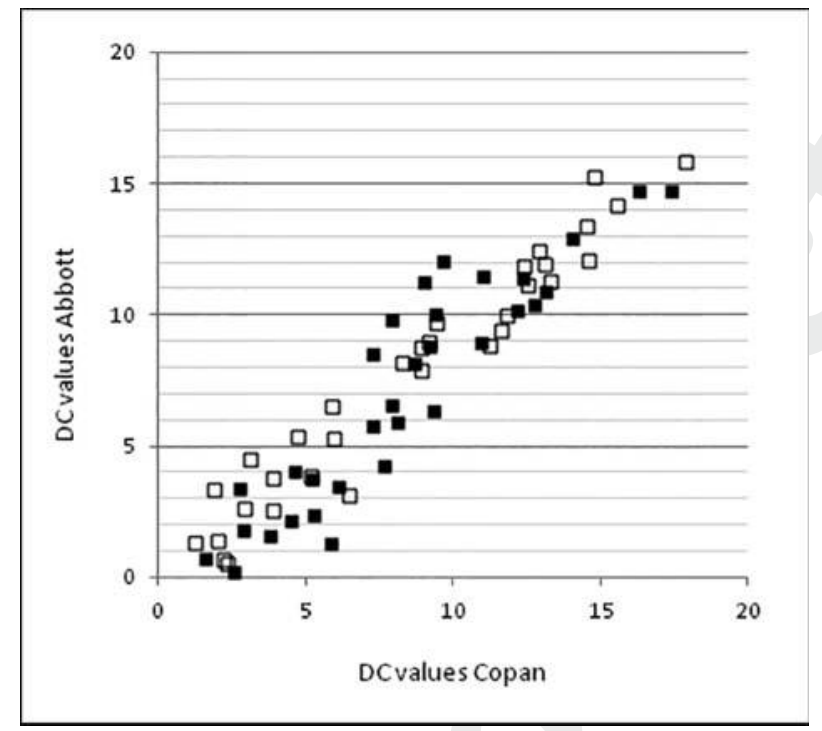

Figure 2 Graphical outline of the DC values of the samples both positive with Abbott and Copan ( $\square$ Abbott swab collected before Copan swab; $\square$ Copan swab collected before Abbott swab).

The number of errors leading to retesting for each swab is listed in Table 1. During the whole study, the majority of all retests was due to automatical errors by m2000-instruments with the Copan swab (29/34 in the first arm and 35/51 in the second arm). An internal control error was the number one reason for retesting (23/29 in the first arm and 31/35 in the second arm), followed by a beyond cutoff error (6/29 in the first arm and $4 / 35$ in the second arm). Of all 21 Abbott-retests, a beyond cutoff error was the only cause of retesting. The order of sampling seems to influence the error rate, as the
Table 1 Number of errors leading to retesting for each arm of the study and for each swab.

\begin{tabular}{lccccc}
\hline & \multicolumn{2}{c}{ 1st A-2nd C } & & \multicolumn{2}{c}{ 1st C-2nd A } \\
\cline { 2 - 3 } \cline { 5 - 6 } & C & A & & C & A \\
\hline IC-error & $23(0 / 23)$ & None & & $31(2 / 31)$ & None \\
CO-error & $6(1 / 6)$ & $5(1 / 5)$ & $4(2 / 4)$ & $16(4 / 16)$ \\
Sum & $29(1 / 29)$ & $5(1 / 5)$ & $35(4 / 35)$ & $16(4 / 16)$ \\
\hline
\end{tabular}

A: Abbott swab; C: Copan swab; IC: internal control; CO: beyond cutoff; numbers within brackets: combined Abbott/Copan errors.

Abbott swab gave significantly more errors when it was taken after the Copan swab (5 vs 16). However, this increase was less obvious with the Copan swab (29 vs 35).

\section{Discussion}

In our study, we evaluated the interchangeability of Copan FLOQSwabs and Abbott swabs for the subsequent detection of $C$. trachomatis in vaginal samples of 1062 Belgian sex workers. Second, we investigated a possible improvement in detection of C. trachomatis using flocked swabs instead of Abbott swabs in this population. In total, 969 $(85.0 \%)$ of the swabs were negative concordant; 69 $(6.1 \%)$ were positive concordant; $10(0.9 \%)$ were positive on the Copan swab but negative on the Abbott swab $(\mathrm{A}-\mathrm{C}+)$ and two $(0.2 \%)$ were positive on the Abbott swab but negative on the Copan swab $(\mathrm{A}+\mathrm{C}-)$. The preponderance of $\mathrm{A}-\mathrm{C}+$ discordances suggests the higher sensitivity of analysis on Copan swabs. In fact, all discordant samples had a 
weak positive test result. This hypothesis is affirmed by the systematically higher DC values measured on Copan swabs, resulting in a significant difference between samples positive both on Copan and Abbott swabs.

The clinical background of the patients with discordant results was retrieved. Within the $\mathrm{A}-\mathrm{C}+$ group $(n=10)$, two patients were symptomatic and eight were asymptomatic. Of those asymptomatic females, one was recently treated for $C$. trachomatis and four were known to display risk behaviour. Within the $\mathrm{A}+\mathrm{C}-$ group $(n=2)$, one patient was symptomatic and one was asymptomatic. However, as mentioned previously, symptoms are no conditio sine qua non for $C$. trachomatis infections. Differences in $C$. trachomatis load have been associated with the presence of clinical symptoms, the transmissibility and persistence of infection, the risk of developing chronic sequelae and the existence of multidrug resistance. The asymptomatic nature of the disease and risk of severe complications emphasise the critical role of sensitive and reliable diagnostic laboratory methods. ${ }^{14,15}$

When comparing both study arms, comparable numbers needed to be retested due to technical issues, such as internal control errors or beyond cutoff results. Most retests (64/85 or $75.3 \%$ ) were attributed to errors with the Copan swab, with internal control issues being the main reason for retesting $(54 / 64$ or $84.4 \%)$. From a technical point of view, it is plausible to assume a link between the higher retest rate and the higher sensitivity of analysis on the Copan swabs. The discordant results left aside, the prevalence of $C$. trachomatis in our study population was $6.7 \%$, which is comparable but lower than the $7.5 \%$ found in a previous study of the same population in 2005. ${ }^{13}$ This prevalence is lower than recorded in immigrant sex workers in Italy $(11 \%)$ and in registered sex workers in Mexico (14.5\%), though higher than reported in the Czech Republic (5.5\%), in Belgian general practices $(5.0 \%)$ and in general population in Britain (1.5\%) and the Netherlands $(2.9 \%){ }^{13}$ The prevalence found in our study $(6.7 \%)$, combined with the test specifications stated in the package insert of the Abbott RealTime C. trachomatis assay (sensitivity of $95.2 \%$ and specificity of $99.3 \%$ ), results in a PPV of $90.7 \%$. As described in the introduction, this PPV makes the need for confirmation by a second NAAT redundant. $^{6,12}$

An ideal swab design collects many cells and allows for their release into media. Copan FLOQswabs are produced by a flocking process, were short nylon fibre strands are attached to the glued end of moulded plastic applicators of a desired shape. The strands are electrostatically charged and are propelled at high velocity, so that their polar ends strike the adhesive to bond them perpendicular to the surface. This process results in a hydrophilic layer of nylon pile with efficient collection and release of cells and secretions collected during sampling. ${ }^{16}$

The analytical advantage of flocked swabs in the sampling of the urogenital tract has been demonstrated by several authors. For instance, a study compared flocked swabs in universal transport medium from Copan Diagnostics to the swabs and transport tubes from three diagnostic kits for $C$. trachomatis: Aptima Combo 2 (Gen-Probe, San Diego, CA, USA), Amplicor (Roche Molecular Systems, Montreal, QC, Canada) and ProbeTec ET (Becton Dickinson Biosciences, Sparks, MD, USA). They used series of diluted positive mocked C. trachomatis and Neisseria gonorrhoeae samples. The flocked swabs showed an equal to better analytical sensitivity, which may translate to better clinical sensitivity for C. trachomatis and $N$. gonorrhoea. ${ }^{16}$ An improved clinical sensitivity for Trichomonas vaginalis was found for Copan flocked swabs in a study comparing self-collected vaginal dacron swabs using an Aptima collection kit, self-collected vaginal Copan flocked swabs and first-void urine from 530 women. Both swabs were transported individually in Aptima specimen transportation media tubes and T. vaginalis was detected using an Aptima NAAT. Next to the better performance of the flocked swabs, the authors stressed the ease of collection and cost efficiency. ${ }^{17}$ In another study, a dual collection device containing flocked and rayon swabs were used to collect vaginal and cervical samples from 494 women. The swabs were individually transported to the laboratory in a dry tube environment, where they were hydrated and tested for high-risk human papillomavirus (hrHPV) and $C$. trachomatis with a Digene hybrid capture two assay (Qiagen, Valencia, CA, USA) and an in-house RealTime PCR, respectively. The flocked swabs identified more hrHPV and C. trachomatis infections from both sampling sites, thereby suggesting a vaginal collection with a flocked swab to be a viable alternative to cervical swabs in hrHPV and $C$. trachomatis screening programmes. ${ }^{18}$

Next to studies with urogenital samples, several reports with impressive performances have appeared evaluating flocked swabs for respiratory sampling. One study investigated the total respiratory epithelial cell yield in parallel nasopharyngeal and nasal swabs sampled with Copan flocked swabs or Copan rayon swabs taken from 16 healthy volunteers and 61 symptomatic patients. In both groups, a two- to threefold increase in cell yield with the flocked design was counted. The authors emphasise the importance of cell yield for NAAT with respect to intracellular pathogen detection. ${ }^{19}$ Indeed, a 
significant concern with regard to the performance of NAAT has been the cellular adequacy of the cervical specimen, since $C$. trachomatis is an intracellular organism. The presence or absence of cells has been shown to significantly affect the sensitivity and the specificity of $C$. trachomatis detection. However, several studies found that samples can test positive for C. trachomatis in the absence of cells or cellular DNA as well, due to extracellular bacteria. ${ }^{16,20-22}$ Other authors found a nasal Copan flocked swab to surpass traditional nasal aspirates in terms of PCR-based virus detection. Moreover, clinicians found flocked swabs easier to evaluate, store and transport. ${ }^{23}$ Similar experiments were set up, comparing nasal Copan flocked swabs with nasopharyngeal aspirates (NPAs) taken from 455 children for the detection of respiratory viruses. Although no statistically significant differences were established, the authors conclude that the excellent sensitivity and specificity of the flocked swabs, complemented by the superb feedback from the nursing staff make them a valid alternative. ${ }^{24}$ Another group compared the viral diagnostic yield for respiratory viruses from Copan flocked swabs with NPAs collected from 196 children tested by RT-PCR. Although the authors consider NPAs as the best specimen, more than acceptable sensitivities were achieved with the flocked swabs as well. ${ }^{25}$

\section{Conclusions}

Copan FLOQSwabs can be used interchangeably with Abbott swabs for Abbott RealTime C. trachomatis PCR. With the Copan swabs, positivity was measured in eight Abbott-negative samples, whilst two samples were only positive with the Abbott swabs. All of these discordant samples were in the low analytical test range. Technical issues lead to retesting of 64 Copan swabs versus 21 Abbott swabs. Thus, while appearing to have an advantage in detecting more positive samples, the use of Copan swabs led to a higher retesting rate, due to technical errors.

\section{Disclaimer Statements}

\section{Contributors}

Funding There is no funding for this article.

Conflict of interest The authors have no conflict of interest.

\section{Ethics approval}

\section{References}

1 Bébéar C, de Barbeyrac B. Genital Chlamydia trachomatis infections. Clin Microbiol Infect. 2009;15:4-10.

2 Available from: http://www.cdc.gov/std/stats13/chlamydia.htm

3 European Centre for Disease Prevention and Control. Sexually transmitted infections in Europe. 2011.
4 Verbrugge R, Moreels S, Crucitti T, et al. Seksueel Overdraagbare Aandoeningen bij de Algemene Bevolking. Gegevens van 2013 voor België en de 3 Regio's. Brussel: WIV-ISP; 2014.

5 Le Roy C, Papaxanthos A, Liesenfeld O, Mehats V, Clerc M, Bébéar C, et al. Swabs (dry or collected in universal transport medium) and semen can be used for the detection of Chlamydia trachomatis using the cobas 4800 system. J Med Microbiol. 2013;62:217-22.

6 Schachter J, Hook EW, Martin DH, Willis D, Fine P, Fuller D, et al. Confirming positive results of nucleic acid amplification tests (NAATs) for Chlamydia trachomatis: all NAATs are not created equal. J Clin Microbiol. 2005;43:1372-3.

7 Castriciano S, Luinstra K, Jang D, Patel J, Mahony J, Kapala J, et al. Accuracy of results obtained by performing a second ligase chain reaction assay and PCR analysis on urine samples with positive or near-cutoff results in the LCx test for Chlamydia trachomatis. J Clin Microbiol. 2002;40:2632-4.

8 Culler EE, Caliendo AM, Nolte FS. Reproducibility of positive test results in the BDProbeTec ET system for detection of Chlamydia trachomatis and Neisseria gonorrhoeae. J Clin Microbiol. 2003;41:3911-4.

9 Gronowski AM, Copper S, Baorto D, Murray PR. Reproducibility problems with the Abbott laboratories LCx assay for Chlamydia trachomatis and Neisseria gonorrhoeae. J Clin Microbiol. 2000;38:2416-8.

10 Peterson EM, Darrow V, Blanding J, Aarnaes S, de la Maza LM. Reproducibility problems with the AMPLICOR PCR Chlamydia trachomatis test. J Clin Microbiol. 1997;35:957-95.

11 Verkooyen RP, Noordhoek GT, Klapper PE, Reid J, Schirm J, Cleator GM, et al. Reliability of nucleic acid amplification methods for detection of Chlamydia trachomatis in urine: results of the first international collaborative quality control study among 96 laboratories. J Clin Microbiol. 2003;41:3013-6.

12 Johnson RE, Newhall WJ, Papp JR, Knapp JS, Black CM, Gift TL, et al. Screening tests to detect Chlamydia trachomatis and Neisseria gonorrhoeae infections - 2002. MMWR Recomm Rep. 2002;51:1-38.

13 Mak RP, Van Renterghem L, Traen A. Chlamydia trachomatis in female sex workers in Belgium: 1998-2003. Sex Transm Infect. 2005;81:89-90.

14 Geisler WM, Suchland RJ, Whittington WL, Stamm WE. Quantitative culture of Chlamydia trachomatis: relationship of inclusion forming units produced in culture to clinical manifestations and acute inflammation in urogenital disease. J Infect Dis. 2001;184:1350-4.

15 Horner P. The case for further treatment studies of uncomplicated genital Chlamydia trachomatis infection. Sex Transm Infect. 2006;82(4):340-3.

16 Chernesky M, Castriciano S, Jang D, Smieja M. Use of flocked swabs and a universal transport medium to enhance molecular detection of Chlamydia trachomatis and Neisseria gonorrhoeae. J Clin Microbiol. 2006;44:1084-6.

17 Jang D, Gilchrist J, Portillo E, Smieja M, Toor R, Chernesky M. Comparison of dacron and nylon-flocked self-collected vaginal swabs and urine for the detection of Trichomonas vaginalis using analyte-specific reagents in a transcription-mediated amplification assay. Sex Transm Infect. 2012;88:160-2.

18 Krech T, Castriciano S, Jang D, Smieja M, Enders G, Chernesky M. Detection of high risk HPV and Chlamydia trachomatis in vaginal and cervical samples collected with flocked nylon and wrapped rayon dual swabs transported in dry tubes. J Virol Methods. 2009;162:291-3.

19 Daley P, Castriciano S, Chernesky M, Smieja M. Comparison of flocked and rayon swabs for collection of respiratory epithelial cells from uninfected volunteers and symptomatic patients. J Clin Microbiol. 2006;44:2265-7.

20 Coutlée F, de Ladurantaye M, Tremblay C, Vincelette J, Labrecque L, Roger M. An important proportion of genital samples submitted for Chlamydia trachomatis detection by PCR contain small amounts of cellular DNA as measured by beta-globin gene amplification. J Clin Microbiol. 2000;38:2512-5

21 Kellogg JA, Seiple JW, Klinedinst JL, Stroll ES, Cavanaugh SH. Improved PCR detection of Chlamydia trachomatis by using an altered method of specimen transport and high-quality endocervical specimens. J Clin Microbiol. 1995;33(10):2765-7.

22 Welsh LE, Quinn TC, Gaydos CA. Influence of endocervical specimen adequacy on PCR and direct fluorescent-antibody 
staining for detection of Chlamydia trachomatis infections. J Clin Microbiol. 1997;35:3078-81.

23 Walsh P, Overmyer CL, Pham K, Michaelson S, Gofman L, DeSalvia L, et al. Comparison of respiratory virus detection rates for infants and toddlers by use of flocked swabs, saline aspirates, and saline aspirates mixed in universal transport medium for room temperature storage and shipping. J Clin Microbiol. 2008;46:2374-6.
24 Abu-Diab A, Azzeh M, Ghneim R, Ghneim R, Zoughbi M, Turkuman S, et al. Comparison between pernasal flocked swabs and nasopharyngeal aspirates for detection of common respiratory viruses in samples from children. J Clin Microbiol. 2008;46:2414-7.

25 Chan KH, Peiris JS, Lim W, Nicholls JM, Chiu SS. Comparison of nasopharyngeal flocked swabs and aspirates for rapid diagnosis of respiratory viruses in children. J Clin Virol. 2008;42:65-9. 